Supporting Information

\title{
Streamlined Mesoporous Silica Nanoparticles with Tunable Curvature from Interfacial Dynamic-Migration Strategy for Nanomotors
}

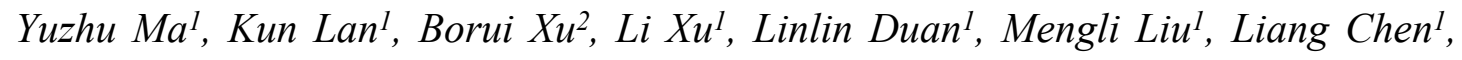

Tiancong Zhao ${ }^{1}$, Jun-Ye Zhang ${ }^{1}$, Zirui Lv ${ }^{1}$, Ahmed A. Elzatahry ${ }^{3}$, Xiaomin Li ${ }^{1 *}$,

Dongyuan Zhao $^{1 *}$

${ }^{1}$ Department of Chemistry, Shanghai Key Laboratory of Molecular Catalysis and Innovative Materials, Laboratory of Advanced Materials, State Key Laboratory of Molecular Engineering of Polymers, iChEM (Collaborative Innovation Center of Chemistry for Energy Materials), Fudan University, Shanghai, 200433, P. R. China.

${ }^{2}$ Department of Materials Science, State Key Laboratory of ASIC and Systems, Fudan University, 220 Handan Road, Shanghai 200433, China

${ }^{3}$ College of Arts and Sciences, Qatar University, PO Box 2713, Doha, Qatar *Corresponding Authors: lixm@fudan.edu.cn; dyzhao@fudan.edu.cn

\section{Content:}

1. Materials and Methods

2. Figures, supplementary text

3. Reference 


\section{Materials and Methods}

Behenyltrimethylammonium bromide (BTAB), octadecytrimethylammonium bromide (STAB), hexadecyltrimethylammonium bromide (CTAB), dodecyltrimethylammonium bromide (DTAB), decyltrimethylammonium bromide ( $\mathrm{D}_{\mathrm{e}} \mathrm{TAB}$ ), polyvinylpyrrolidone (PVP, molecular weight 55,000 $\mathrm{g} \mathrm{mol}^{-1}$ ), sodium citrate monohydrate (99\%) and sodium citrate dihydrate (99\%), tetraethyl orthosilicate (TEOS), were purchased from Aladdin (Shanghai, China). $n$-Pentanol, ammonium hydroxide (28 wt\%), anhydrous ethanol, iron (III) chloride hexahydrate (99\%) iron (II) chloride tetrahydrate (99.95\%) were purchased from Sinopharm (Shanghai, China). All chemicals were used as received without further purification. Deionized water was used for all experiments.

\section{Synthesis}

The streamlined hollow mesoporous silica nanotadpoles were synthesized using an interfacial dynamic-migration strategy. Typically, $40.0 \mathrm{mg}$ of hexadecyltrimethylammonium bromide $(\mathrm{CTAB})$ and $1.0 \mathrm{~g}$ of polyvinylpyrrolidone (PVP) were dissolved in $10.0 \mathrm{~mL}$ of $n$-pentanol under sonication for $2 \mathrm{~h}$. Then, 1.00 $\mathrm{mL}$ of ethanol, $0.20 \mathrm{~mL}$ of deionized water containing $0.020 \mathrm{~mL}$ of aqueous sodium citrate solution $(0.18 \mathrm{M})$ and $0.10 \mathrm{~mL}$ of $\mathrm{NH}_{3} \cdot \mathrm{H}_{2} \mathrm{O}(28 \mathrm{wt} \%)$ were added to the above mixture in sequence. After sonicating for $0.5 \mathrm{~h}, 0.54 \mathrm{mmol}$ of tetraethyl orthosilicate (TEOS) was added. The streamlined mesoporous silica nanotadpoles with an open cavity (tail length: $\sim 160 \mathrm{~nm}$ ) could be formed after standing for $1.5 \mathrm{~h}$ at room temperature, and further extending the reaction time to $10 \mathrm{~h}$, the silica nanotadpoles with enclosed cavity and elongated tail $(\sim 295 \mathrm{~nm})$ could be formed. The final products were centrifuged and washed for three times with ethanol. Then, the collected products were extracted with $0.1 \mathrm{M}$ of $\mathrm{HCl}$ ethanol solution at $60{ }^{\circ} \mathrm{C}$ for $12 \mathrm{~h}$ twice to remove the residual surfactants.

For regulating the curvature of the streamlined nanoparticles, the above reactions were performed similarly except that replacing CTAB with behenyltrimethylammonium bromide (BTAB), octadecytrimethylammonium bromide (STAB), dodecyltrimethylammonium bromide (DTAB), and decyltrimethylammonium bromide $\left(\mathrm{D}_{\mathrm{e}} \mathrm{TAB}\right)$

\section{Loading of $\mathrm{Fe}_{3} \mathrm{O}_{4}$ nanoparticles}

Hydrophilic $\mathrm{Fe}_{3} \mathrm{O}_{4}$ nanoparticles were prepared based on the method reported previously in the literature. ${ }^{1}$ In brief, $0.40 \mathrm{~g}$ of $\mathrm{FeCl}_{2} \cdot 4 \mathrm{H}_{2} \mathrm{O}$ and $1.70 \mathrm{~g}$ of $\mathrm{FeCl}_{3} \cdot 6 \mathrm{H}_{2} \mathrm{O}$ were added to $20.0 \mathrm{~mL}$ water in a three-necked flask and heated to $90{ }^{\circ} \mathrm{C}$ under nitrogen atmosphere. After adding $2.50 \mathrm{~mL}$ of ammonia and stirring for $0.5 \mathrm{~h}, 1.0 \mathrm{~g}$ of sodium citrate monohydrate dissolved in $2.0 \mathrm{~mL}$ of water was added to the above solution, and the reaction was continued for another $2 \mathrm{~h}$. The $\mathrm{Fe}_{3} \mathrm{O}_{4}$ nanoparticles were washed twice with deionized water and redispersed in $20.0 \mathrm{~mL}$ of deionized water.

For the loading of $\mathrm{Fe}_{3} \mathrm{O}_{4}$ nanoparticles into the mesoporous silica nanotadpoles, $0.020 \mathrm{~mL}$ of the above $\mathrm{Fe}_{3} \mathrm{O}_{4}$ aqueous solution with a concentration of $2.0 \mathrm{mg} / \mathrm{mL}$ was added to $10.0 \mathrm{~mL}$ of $n$-pentanol after dissolving $1.0 \mathrm{mg}$ of PVP and $40.0 \mathrm{mg}$ of CTAB. 
All the other reaction conditions were consistent with that of the synthesis of silica nanotadpoles.

\section{Movement analysis of the nanomotors}

The motions of the nanomotors with different curvatures in varied $\mathrm{H}_{2} \mathrm{O}_{2}$ concentrations were tracked and recorded by NanoSight NS300. The aqueous solution of nanotadpoles with a concentration of $\sim 10^{8}$ particles $\mathrm{mL}^{-1}$ was measured at a temperature of $30^{\circ} \mathrm{C}$. The mean-square-displacement (MSD) was calculated by the formula: $\operatorname{MSD}(\Delta t)=\left\langle\left(x_{\mathrm{i}}\right.\right.$ $\left.\left.(t+\Delta t)-x_{\mathrm{i}}(t)\right)^{2}\right\rangle(i=2)$, where $x$ is a two-dimensional vector and $i$ is an index to show $x$ and $y$. The effective diffusion coefficient $\left(D_{\text {eff }}\right)$ was extracted MSD $=D_{\text {eff }}(4 \Delta t)$. In all cases, at least 15 nanomotors were analyzed.

\section{Simulation of self-diffusiophoresis nanomotor}

COMSOL Multiphysics software was used to simulate the steady-state concentration gradient distribution of nanomotor due to the catalytic reaction of hydrogen peroxide. The model was established on the Transport of Diluted Species interface. Considering that the loading amount of catalyst are basically the same in the three nanomotors, we assumed that the catalytic reaction occurs at the inner surface of head with same reaction rate and area. Therefore, we used inflow boundary condition at the reaction surface which diameter $l$ is fixed as $80 \mathrm{~nm}$, and the concentration is set to $750 \mathrm{~mol} \mathrm{~m}^{-}$ ${ }^{3}{ }^{[2]}$ The $\mathrm{O}_{2}$ molecular diffusion coefficient is set as $2.42 \times 10^{-9} \mathrm{~m}^{2} \mathrm{~s}^{-1}$. The contour of nanomotor was built using elliptic equation for the head and Bézier curve for the body. Thus, the curvature of head was calculated as $a / b^{2}$. Other geometric parameters include body length $L$, opening diameter $D$, wall thickness $d$. The data of concentration gradient was obtained after a running time as $10^{-5} \mathrm{~s}$ and collected at the point near the opening inner wall.

\section{Characterizations}

Nitrogen adsorption-desorption isotherms were performed at $77 \mathrm{~K}$ with a Micromeritcs Tristar 3020 analyzer (USA). The Brunauer-Emmett-Teller (BET) specific surface areas was calculated from the adsorption data in the relative pressure $\left(P / P_{0}\right)$ ranging from 0.5 to 1.0. The pore size distributions and pore volumes were derived from the adsorption branches of the isotherms using the Barrett-Joyner-Halenda (BJH) model. The field-emission scanning electron microscope (FESEM) observations were taken on a Hitachi FE-SEM-4800 microscope operating at $20 \mathrm{kV}$ without any metal coating. Transmission electron microscopy (TEM) measurements were taken on a JEOL JEM$2100 \mathrm{~F}$ microscope (Japan) operated at $200 \mathrm{kV}$. The samples used for TEM and FESEM analyses were dispersed in ethanol and dried on amorphous carbon-coated $\mathrm{Cu}$ grids. The quantitative analyses of the $\mathrm{Fe}_{3} \mathrm{O}_{4}$ loaded in the silica nanotadpoles were determined by inductively coupled plasma (ICP) based on $\mathrm{Fe}^{3+}$ concentration.

\section{Curvature calculation formula}

The surface curvature of the mesoporous particles was calculated from the parabolic model of $y=a x^{2}$. The curvature of parabola at the vertex $(0,0)$ was defined as the 
maximum curvature of the streamlined nanoparticle. So, the curvature radius and surface curvature could be calculated as following:

$$
r=\frac{\left(1+\left(y^{\prime}\right)^{2}\right)^{\frac{3}{2}}}{\left|y^{\prime \prime}\right|}=\frac{1}{2 a}
$$

$$
k=\frac{1}{r}=2 a
$$

Where $r$ is the curvature radius, $k$ is the surface curvature $\left(\mathrm{nm}^{-1}\right), y^{\prime}$ is the first derivative of the model curve, $y^{\prime \prime}$ is the second derivative of the model curve, $a$ is the quadratic coefficient of the parabolic equation. 


\section{Supplementary Figures and Tables}

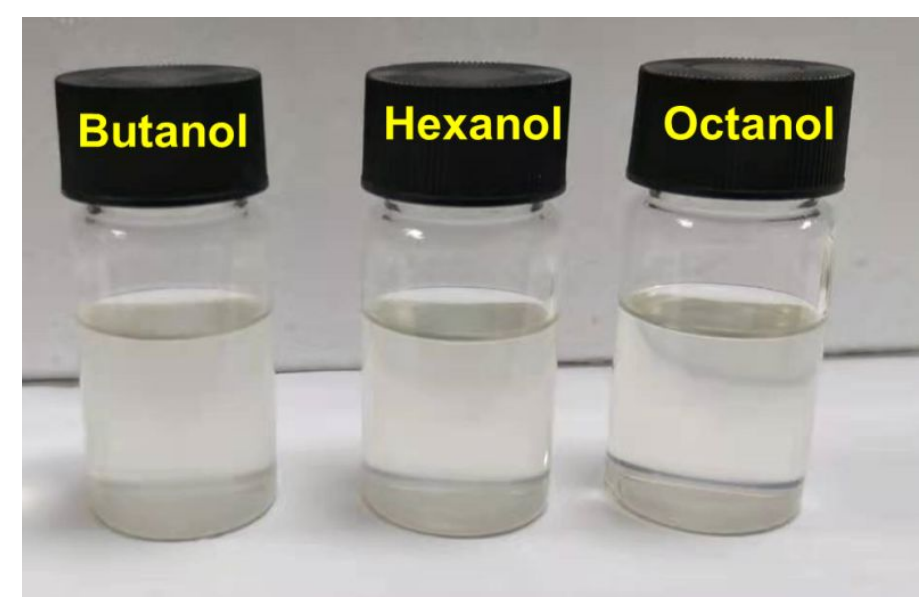

Figure S1. The optical photograph of "water in oil" nanoemulsions formed by using butanol, hexanol and octanol as the oil phase. It can be seen that the clear and stable emulsions without phase separation can be obtained by using other alcohol compounds with different alkyl chain lengths. 


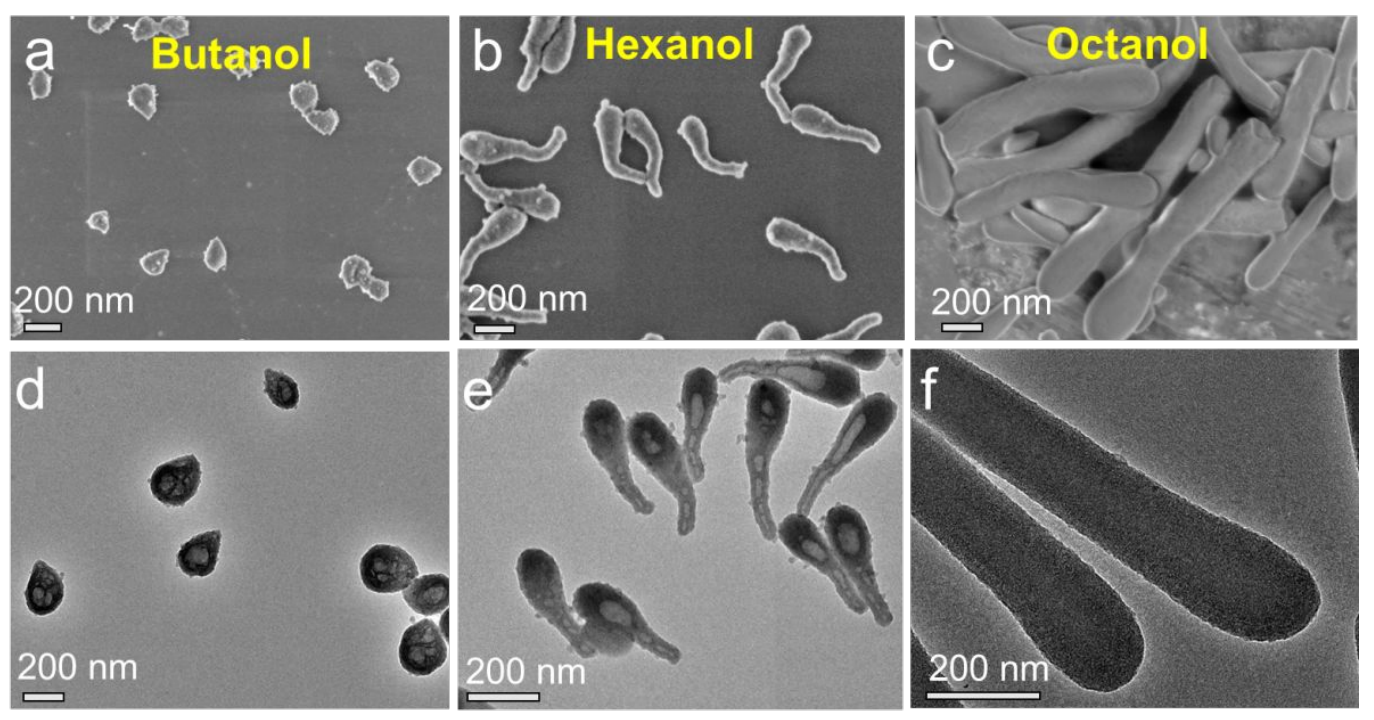

Figure S2. (a-c) SEM and (d-f) TEM images of the mesoporous silica nanoparticles obtained in the nanoemulsion from different alcohol compounds; (a, d) butanol, (b, e) hexanol, and (c, f) octanol. It can be seen that similar streamlined nanoparticles are formed. With the increase of the alkyl chain length of alcohol compounds, the tail lengths of the obtained nanoparticles become longer ( $\sim 115 \mathrm{~nm}$ for butanol, $\sim 277 \mathrm{~nm}$ for hexanol, $\sim 1150 \mathrm{~nm}$ for octanol), while the inner diameter tends to decrease $(\sim 145$ $\mathrm{nm}$ for butanol, $\sim 93 \mathrm{~nm}$ for hexanol, $\sim 72 \mathrm{~nm}$ for octanol). 


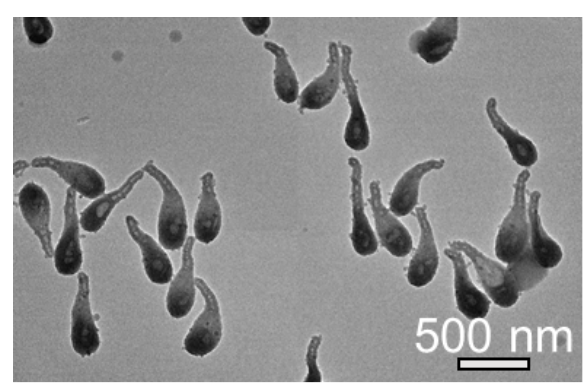

Figure. S3. TEM image of the streamlined tadpole-shaped nanoparticles obtained after being scaled up. It can be seen that this synthesis process still exhibits good repeatability after magnifying the amount of $n$-pentanol in the original system by 10 times (from 10 to $100 \mathrm{~mL}$ ). Meanwhile, the quality of the nanoparticles is as good as that obtained from small batch synthesis. 

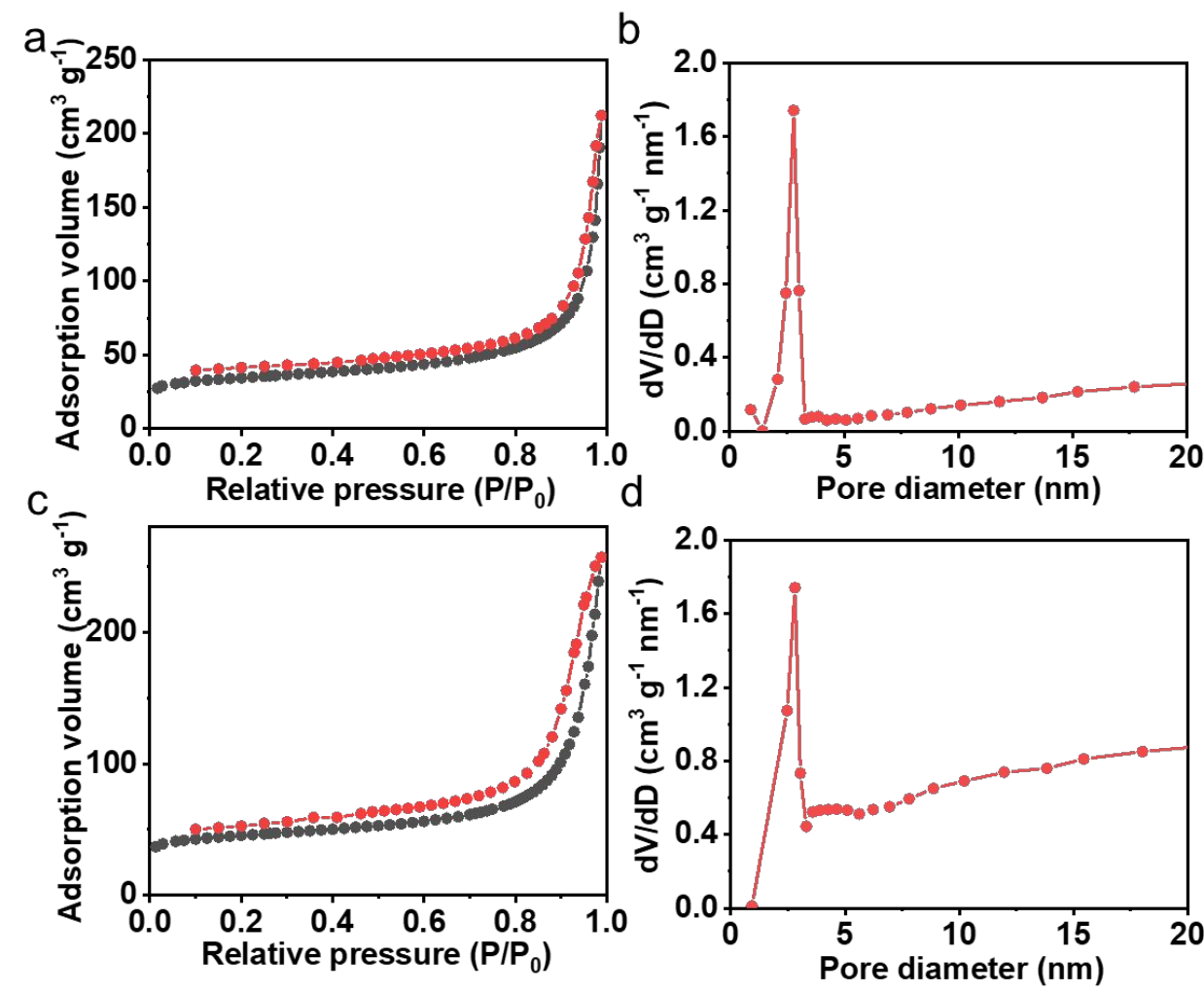

Figure S4. (a, c) Nitrogen sorption isotherms, and (b, d) pore size distribution of the mesoporous silica nanotadpoles; $(a, b)$ nanotadpole-30, and $(c, d)$ nanotadpole-160. It can be seen that the obtained nanotadpole-30 and nanotadpole-160 all display characteristic type-IV curves with distinguishable capillary condensation at $P / P_{0}=0.4$ to 0.9 , indicating the abundant mesopores. The surface areas of the nanotadpole-30 and nanotadpole-160 are measured to be 118 and $256 \mathrm{~m}^{2} \mathrm{~g}^{-1}$, respectively, and the mesopore sizes are mainly concentrated around $2.7 \mathrm{~nm}$. 


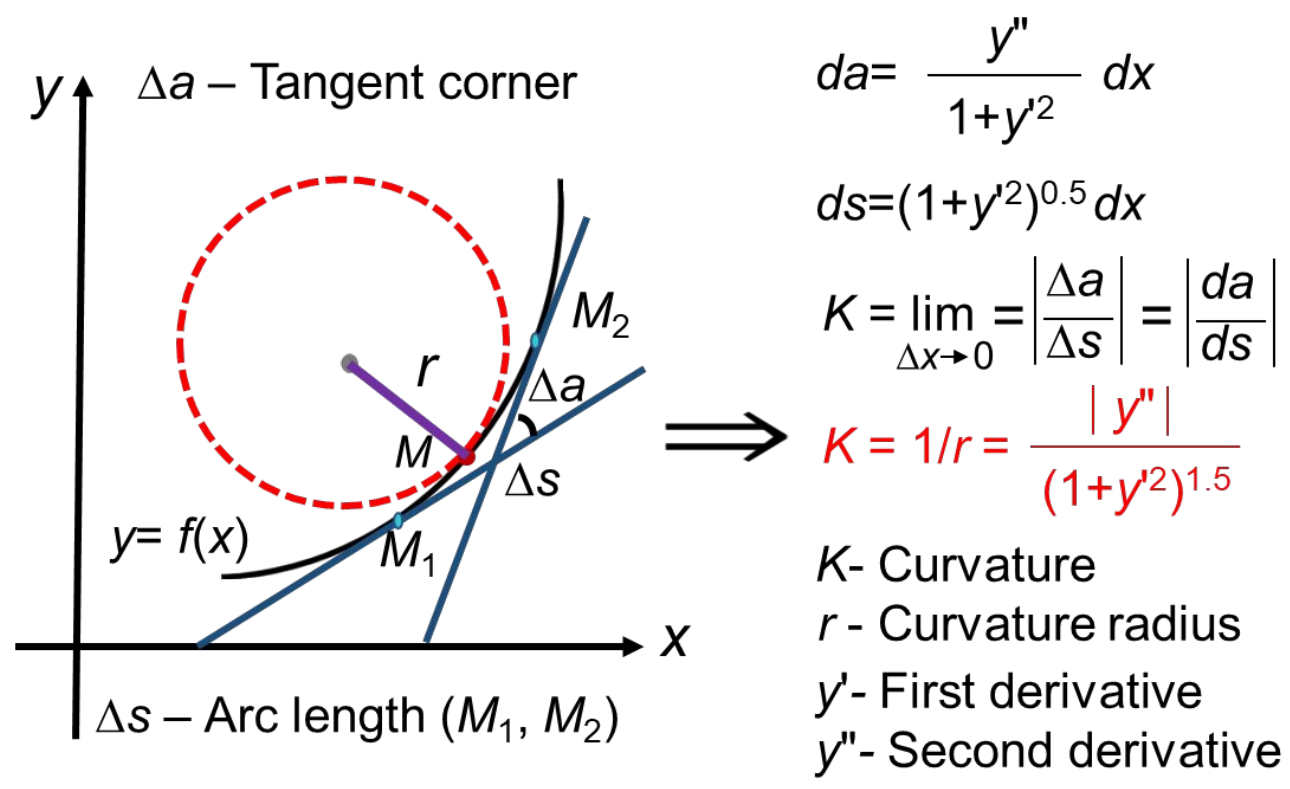

Figure S5. Calculation formula of curvature in mathematics. The curve curvature is the rotation rate of the tangent direction angle to the arc length for a certain point (M) on the curve, which is defined by differentiation as following:

$$
\begin{gathered}
K=\lim _{\Delta x \rightarrow 0}\left|\frac{\Delta \alpha}{\Delta s}\right|=\left|\frac{d \alpha}{d s}\right| \\
K=\frac{1}{r}=\frac{\left|y^{\prime \prime}\right|}{\left(1+y^{\prime 2}\right)^{\frac{3}{2}}}
\end{gathered}
$$

Where $K$ is the surface curvature $\left(\mathrm{nm}^{-1}\right) ; \Delta \alpha$ is the tangent corner, $\Delta \mathrm{s}$ is the arc length between $M_{l}$ and $M_{2}, y^{\prime}$ is the first derivative of the curve; $y^{\prime \prime}$ is the second derivative of the curve. The curvature circle and the curve have a common tangent and curvature at point $\mathrm{M}$.

The curvature radius at point $\mathrm{M}$ is the reciprocal of the curvature of the curve at this point,

$$
r=\frac{1}{K}=\frac{\left(1+y^{\prime 2}\right)^{\frac{3}{2}}}{\left|y^{\prime \prime}\right|}
$$

Where $r$ is the curvature radius (nm). 


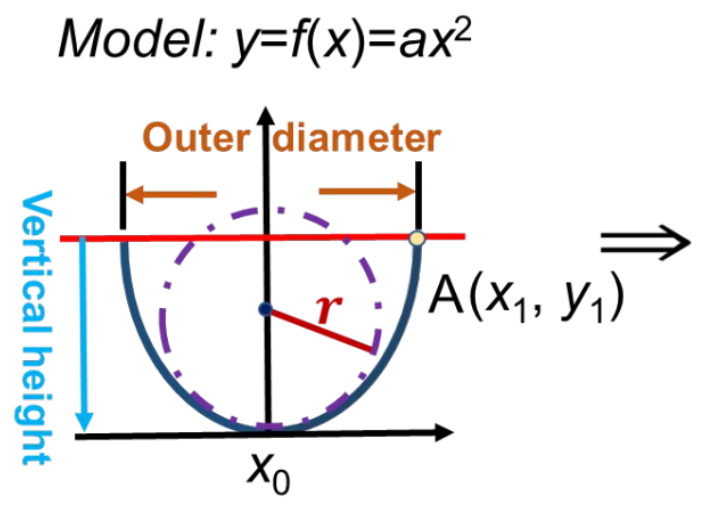

$$
\begin{gathered}
K=1 / r \quad y^{\prime}=2 a x \quad y^{\prime \prime}=2 a \\
K=\frac{\left|y^{\prime \prime}\right|}{\left(1+y^{\prime 2}\right)^{1.5}}=\frac{|2 a|}{\left(1+(2 a x)^{2}\right)^{1.5}} \\
\text { Maximum curvature } \\
x=x_{0}=0 \quad K_{\max }=2 a
\end{gathered}
$$

Figure S6. Calculation of curvature of the streamlined mesoporous silica nanoparticles: Two-dimensional (2-D) coordinate plane curves were introduced to simulate the shape of streamlined nanoparticles. Because the projection of the streamline nanoparticles (the head part) in 2-D plane was similar to a parabola, we used the parabolic equation to simulate the head shape of the streamline nanoparticles,

$$
f(x)=a x^{2}
$$

The curvature of parabola at the vertex $(0,0)$ was defined as the maximum curvature of the streamline nanoparticle. In this case,

$$
\begin{gathered}
y^{\prime}=2 a x \\
y^{\prime \prime}=2 a \\
x_{0}=0
\end{gathered}
$$

So, the head curvature of the streamline nanoparticle was

$$
K=\frac{\left|y^{\prime \prime}\right|}{\left(1+y^{\prime 2}\right)^{\frac{3}{2}}}=\frac{|2 a|}{\left(1+(2 a x)^{2}\right)^{\frac{3}{2}}}=2 a
$$

The quadratic coefficient $a$ could be obtained by putting the coordinates of point A ( $x_{1}$, $y_{1}$ ) into the parabolic equation, where $x_{1}$ is half of the outer diameter of the streamlined nanoparticle, $y_{1}$ is the vertical height at point $\mathrm{A}$. 

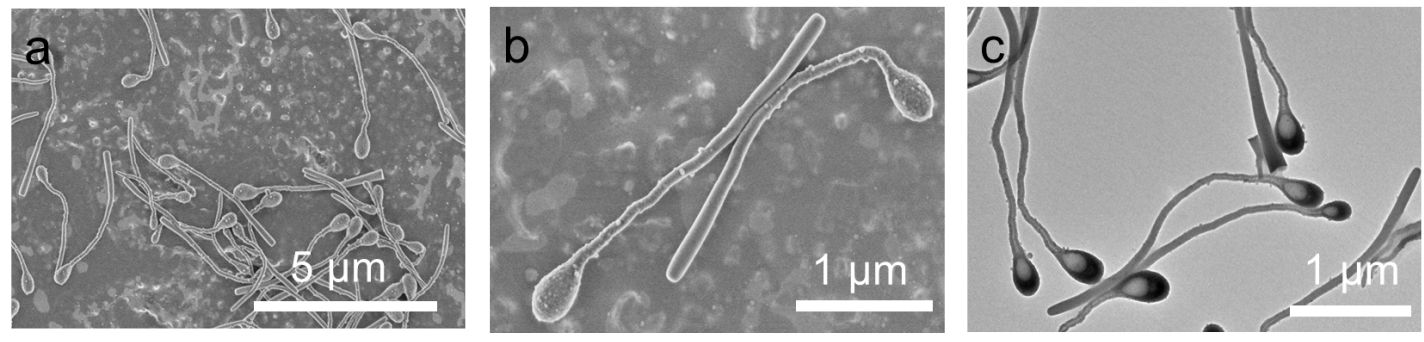

Figure S7. (a, b) SEM and (c) TEM images of the obtained tertiary structured 1-D nanowires with the increase of the water volume, showing that the tertiary structured 1D nanowires with a length of 2-4 $\mu \mathrm{m}$ can be achieved when the water volume is increased to $10 \%$. The silica nanowires are composed by a streamlined head with an outer diameter of $\sim 185 \mathrm{~nm}$, slender nanotube and nanorod with a diameter of $\sim 95 \mathrm{~nm}$. 


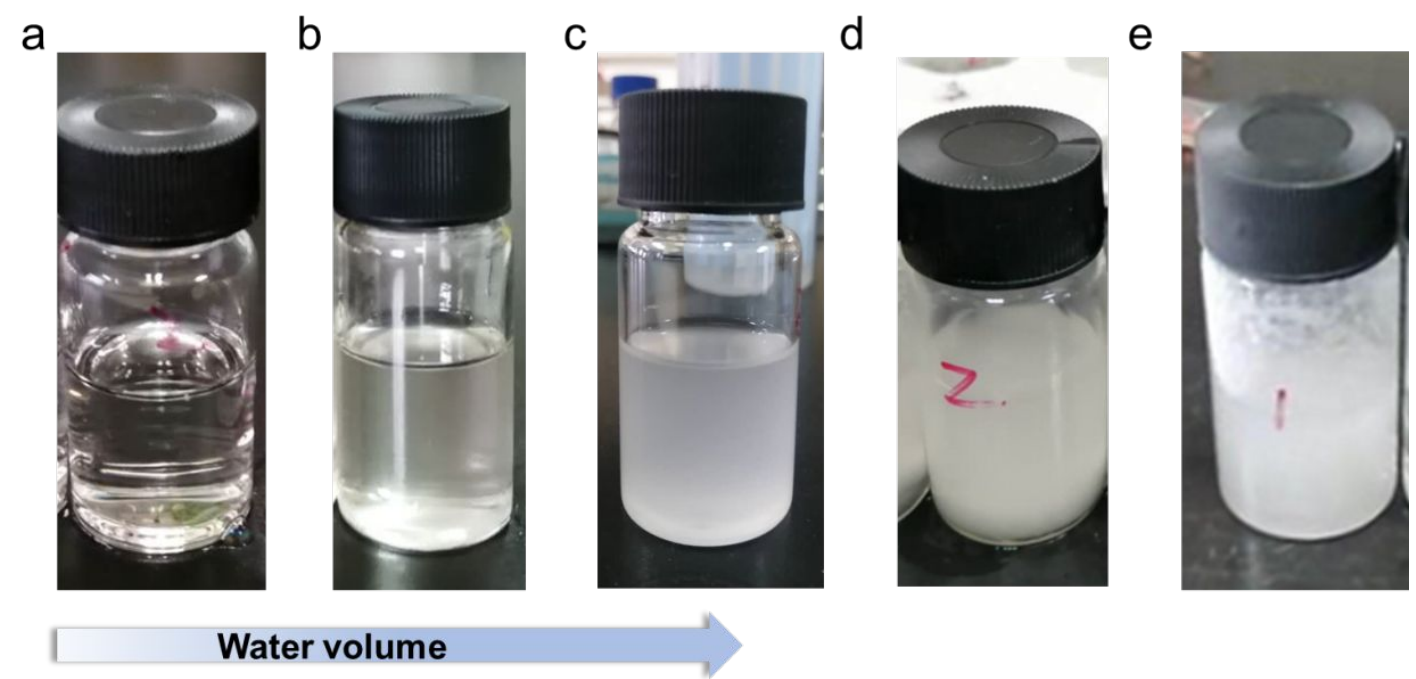

Figure S8. The photograph of the emulsion solutions obtained by regulating the volume fraction of water: (a) $3 \%$; (b) $5 \%$; (c) $8 \%$; (d) $10 \%$; (e) $20 \%$, showing that the turbidities of the emulsions increased as the water volume. 

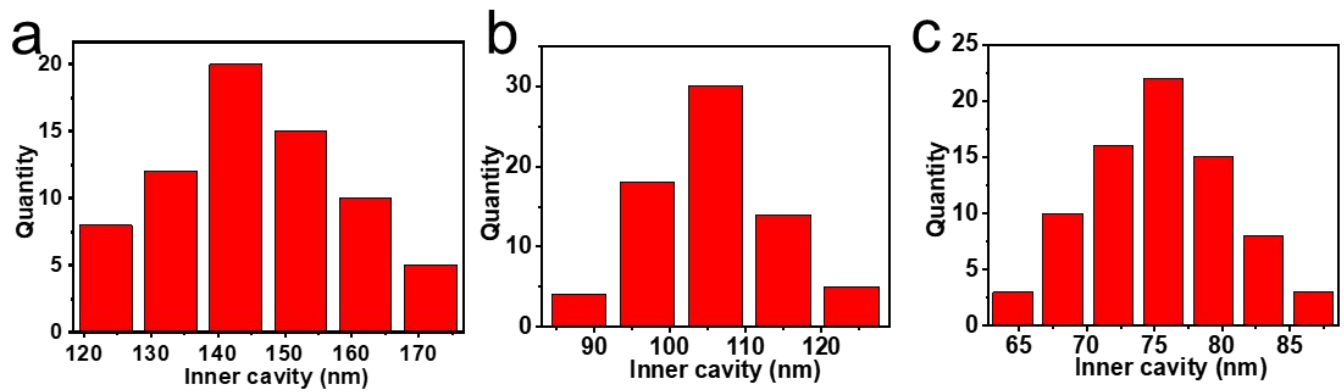

Figure S9. The distribution histograms of the inner cavity sizes of the streamlined mesoporous silica nanoparticles prepared by regulating the volume fraction of water: (a) $3 \%$; (b) $5 \%$; and (c) $8 \%$. It can be seen that the diameter of the inner cavity at the head part of the nanoparticles shrinks from $\sim 145$ to $\sim 77 \mathrm{~nm}$ as the water volume increases, while the curvature of these samples retains unchanged. 


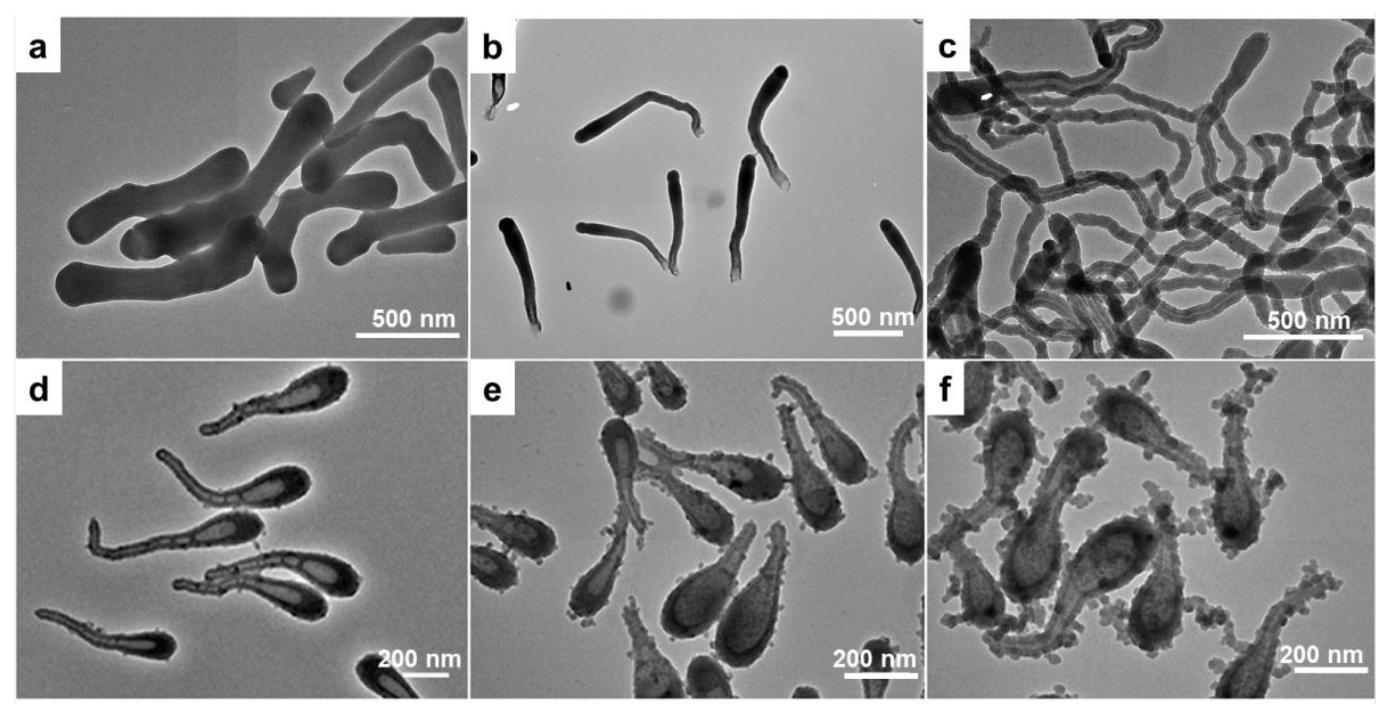

Figure S10. TEM images of the samples obtained with different concentrations of surfactant CTAB: (a) $0 \mathrm{mmol} / \mathrm{mL}$; (b) $2.38 \mathrm{mmol} / \mathrm{mL}$; (c) $4.77 \mathrm{mmol} / \mathrm{mL}$; (d) 9.54 $\mathrm{mmol} / \mathrm{mL}$; (e) $14.32 \mathrm{mmol} / \mathrm{mL}$, and (f) $19.09 \mathrm{mmol} / \mathrm{mL}$. These results show that the $\mathrm{CTAB}$ concentration plays a key role in the formation of the cavity and the streamline structure. Without $\mathrm{CTAB}$, non-porous vimineous solid silica nanorods were formed (Figure S10a). An elongated tubular structure appears when the concentration of surfactant is less than $7.16 \mathrm{mmol} / \mathrm{mL}$ (Figure S10b, c). The streamlined mesoporous silica nanotadpoles with a large cavity could be formed at a surfactant concentration of $9.54 \mathrm{mmol} / \mathrm{mL}$ (Figure S10d). However, the phase separation occurs when the surfactant is excessive (more than $13.13 \mathrm{mmol} / \mathrm{mL}$ ), resulting in the formation of many small silica particles attached on the surface of the mesoporous silica nanotadpoles (Figure S10e, f). 

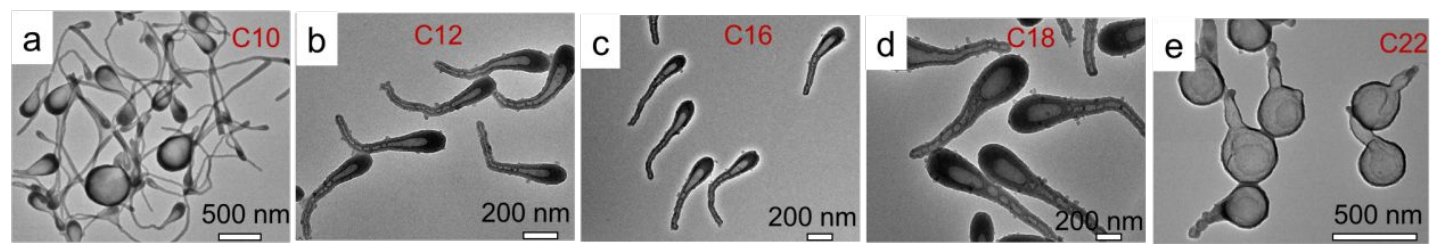

Figure S11. TEM images of the mesoporous silica nanoparticles synthesized by using the cationic surfactants with different alkyl chain lengths: (a) DeTAB, C10, (b) DTAB, C12, (c) CTAB, C16, (d) STAB, C18, and (e) BTAB, C22. When the alkyl chain length of the surfactant is relatively short $\left(\mathrm{D}_{\mathrm{e}} \mathrm{TAB}, \mathrm{C} 10\right)$, the droplets became unstable and the curvature of the obtained nanoparticles was not uniform (curvature value: 2.42$\left.6.67 \times 10^{-2} \mathrm{~nm}^{-1}\right)$. However, as the alkyl chain length increased to $\mathrm{C} 22$, the stretching effect of the hydrophobic chain in the oil phase became stronger, so that the nanoparticles were basically retained the original curvature of the droplets, thus losing the streamlined structure (curvature value: $6.67 \times 10^{-2} \mathrm{~nm}^{-1}$ ). With the gradual increase of the alkyl chain length from $\mathrm{C} 12$ to $\mathrm{C} 18$, the obtained mesoporous silica nanotadpoles present streamlined structure with the curvature gradually decreases from $5.56 \times 10^{-2}$ to $2.96 \times 10^{-2} \mathrm{~nm}^{-1}$. 

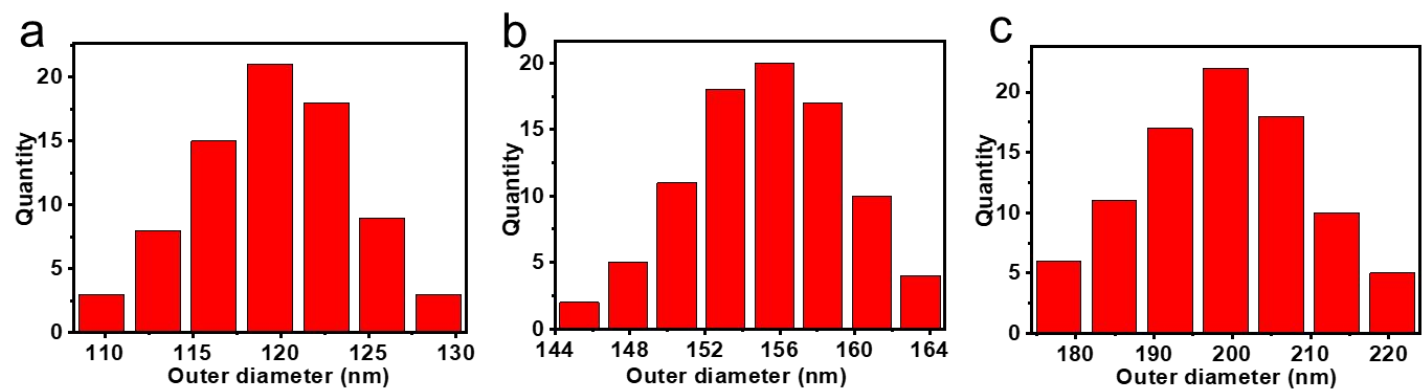

Figure S12. The distribution histograms of the outer diameters of the mesoporous silica nanoparticles with different curvatures obtained by using surfactants with different alkyl chain lengths: (a) DTAB, C12, (b) CTAB, C16, and (c) STAB, C18. It can be intuitively observed that with the increase of alkyl chain length, the outer diameter of the nanoparticles also increases from $\sim 122$ to $\sim 205 \mathrm{~nm}$. 


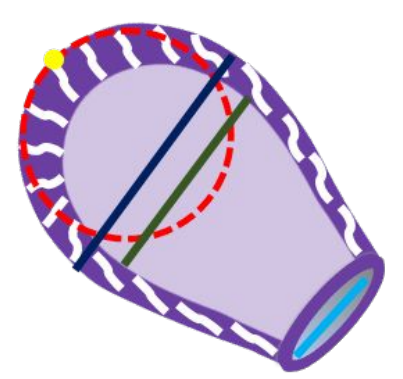

- Opening Curvature circle

— Outer diameter — Inner diameter

Figure S13. Structural model and the related parameters of the silica nanotadpoles. 

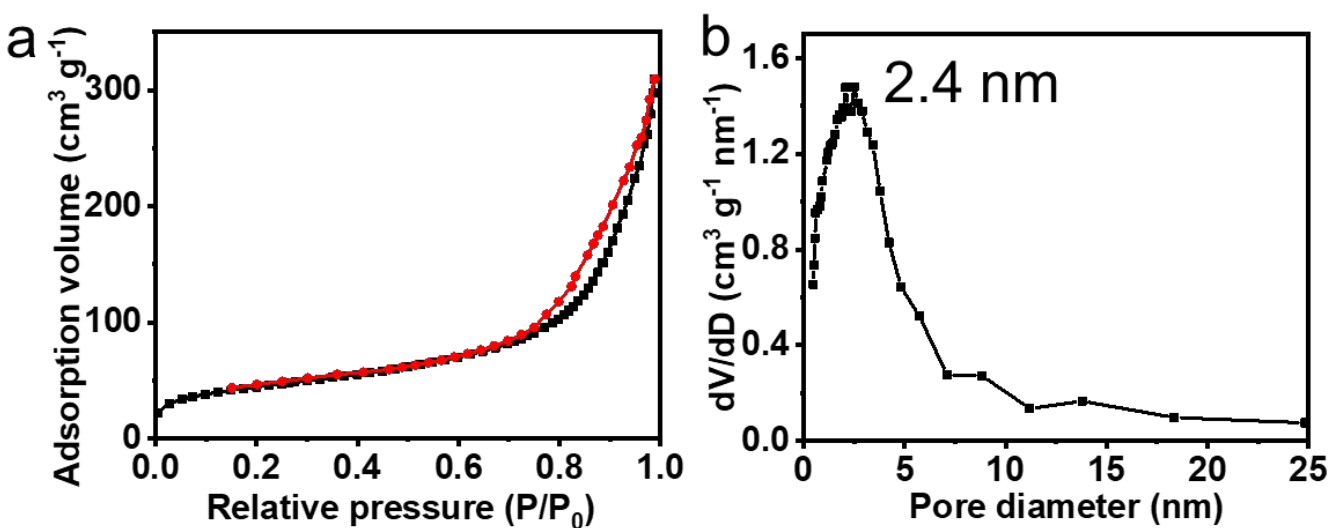

C
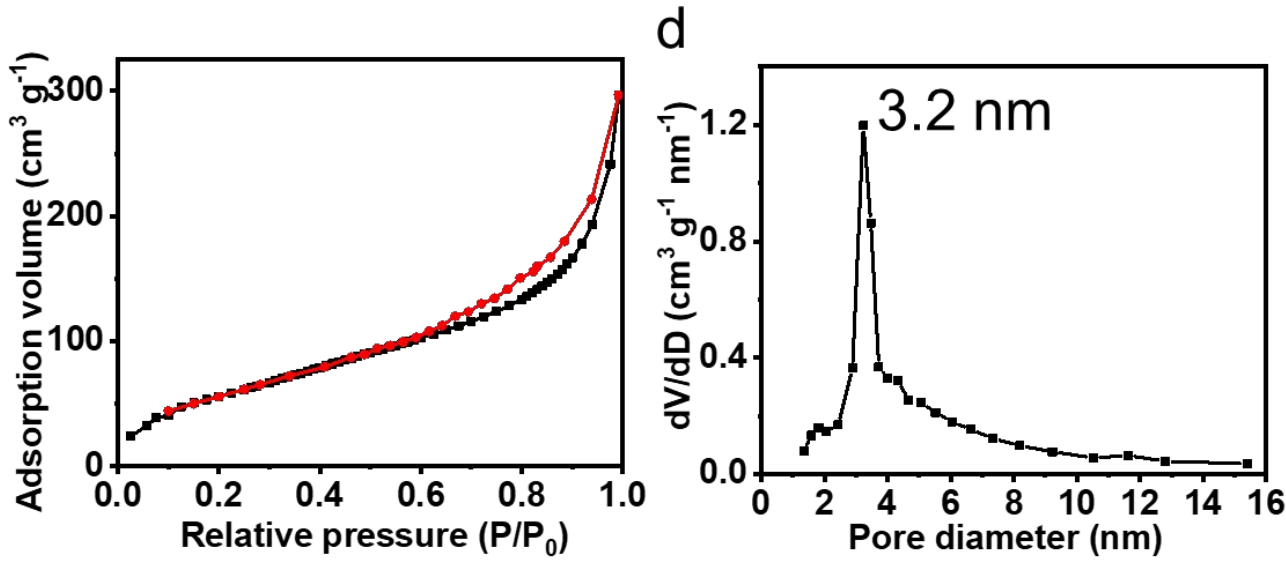

Figure S14. The nitrogen sorption isotherms and pore size distribution of the mesoporous silica nanoparticles synthesized by using the surfactants with different alkyl chain lengths: $(\mathrm{a}, \mathrm{b})$ DTAB with $\mathrm{C} 12$; $(\mathrm{c}, \mathrm{d}) \mathrm{STAB}$ with $\mathrm{C} 18$. It can be seen that all the hollow mesoporous silica nanotadpoles display characteristic type-IV curves with distinguishable capillary condensation at $P / P_{0}=0.6$ to 0.9 (Figure S14a, c), indicating the abundant open mesopore channels. The BET surface areas are calculated to be 488 and $424 \mathrm{~m}^{2} \mathrm{~g}^{-1}$, respectively. With the increase of the alkyl chain length, the pore size of the obtained mesoporous silica nanotadpoles also increases from 2.4 to 3.2 nm. (Figure S14b, d) 

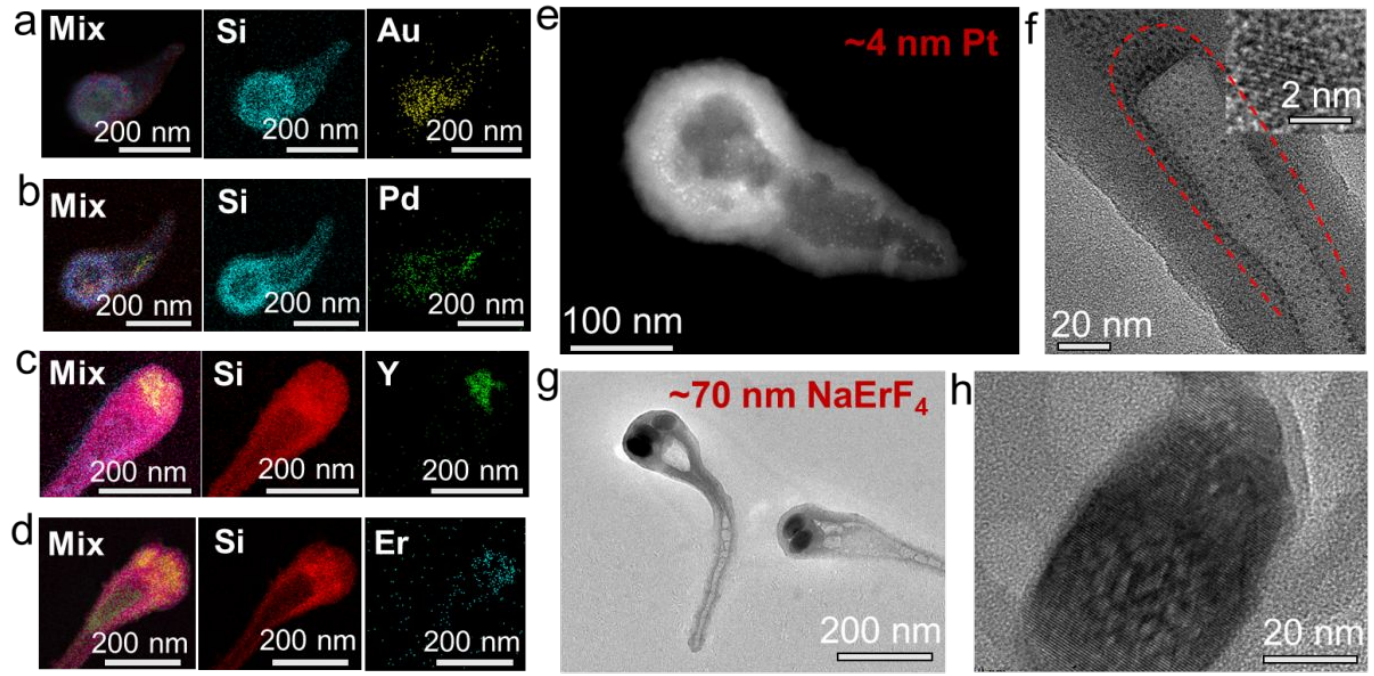

$200 \mathrm{~nm}$

Figure S15. (a-d) Element mappings of the streamlined nanotadpoles loaded with different hydrophilic nanoparticles in the cavity; (a) Au nanocrystals, (b) Pd nanocrystals, (c) $\mathrm{NaYF}_{4}$ nanocrystals, (d) $\mathrm{NaErF}_{4}$ nanocrystals; (e) STEM and (f-h) TEM images of the silica nanotadpoles loaded with $(\mathrm{e}, \mathrm{f})$ the Pt nanocrystals $(\sim 4 \mathrm{~nm})$, and $(\mathrm{g}, \mathrm{h}) \mathrm{NaErF}_{4}$ nanocrystals $(\sim 70 \mathrm{~nm})$. The inset (f) is the high-resolution TEM image of the Pt nanocrystals. The results show that the position of the functional nanocrystals in the cavity is very relevant to the size of the nanoparticles. The smaller nanocrystals tend to disperse in the whole cavity, while the larger nanocrystals tend to deposit at the head of the cavity. We speculate that the variation of the nanoparticles' distribution in cavity results from the different interface dynamic-migration capabilities of the different sized nanoparticles. The larger the particle size is, more difficult the functional nanoparticles are to migrate with the oil-water interface. 

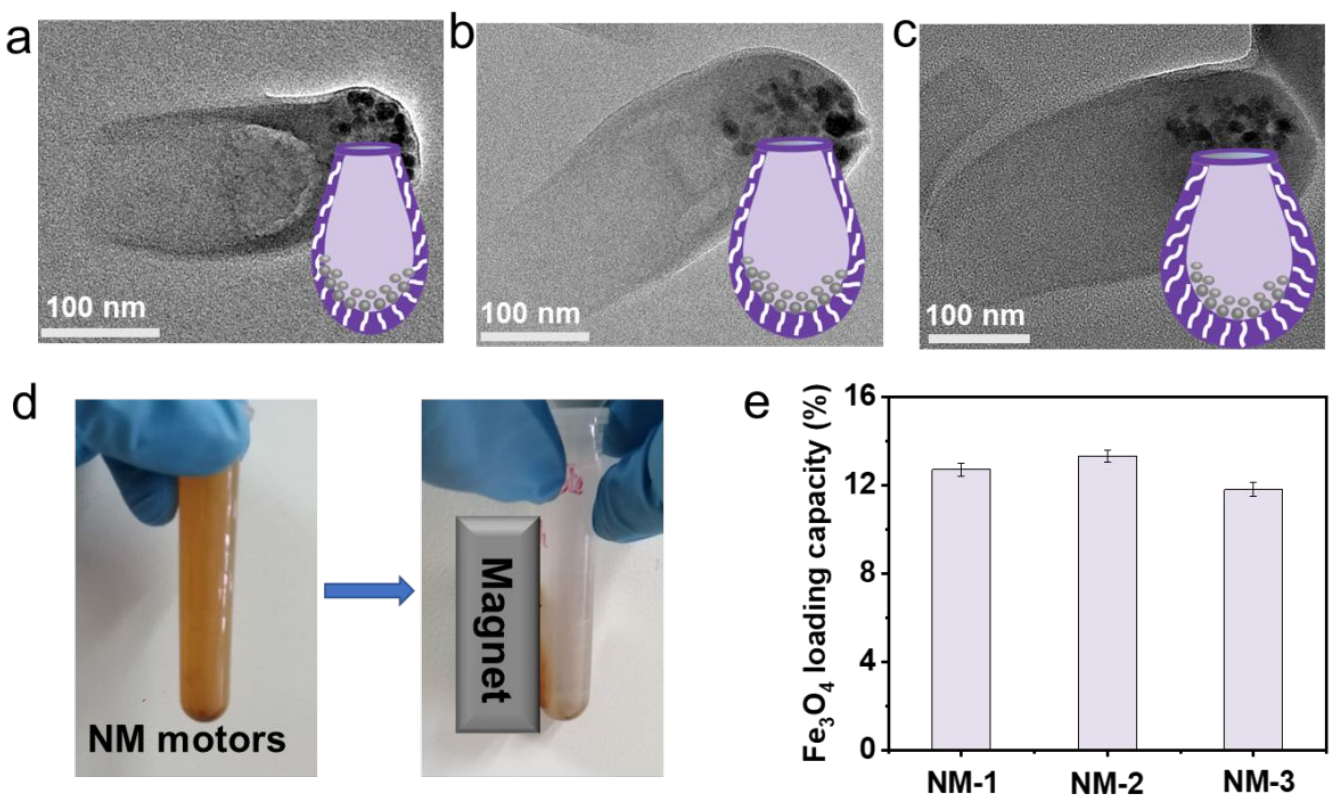

Figure S16. (a-c) TEM images of the $\mathrm{Fe}_{3} \mathrm{O}_{4}$-loaded mesoporous silica nanomotors with different surface curvatures (a) $5.56 \times 10^{-2}$, (b) $3.76 \times 10^{-2}$, (c) $2.96 \times 10^{-2} \mathrm{~nm}^{-1}$; (d) The collection of the $\mathrm{Fe}_{3} \mathrm{O}_{4}$-loaded streamlined nanomotors using a magnet. (e) The loading capacities of the $\mathrm{Fe}_{3} \mathrm{O}_{4}$ catalysts in the three nanomotors. NM-1: $12.74 \%$; NM-2: $\sim 13.31 \%$; NM-3: $\sim 11.82 \%$. It can be seen that the tadpole shape is retained very well after loading $\mathrm{Fe}_{3} \mathrm{O}_{4}$ nanoparticles. $\mathrm{The} \mathrm{Fe}_{3} \mathrm{O}_{4}$ nanoparticles are mainly deposited at the bottom of the nanotadpoles. The $\mathrm{Fe}_{3} \mathrm{O}_{4}$-loaded nanomotors can be collected in $30 \mathrm{~s}$ using a magnet. The loading capacities of $\mathrm{Fe}_{3} \mathrm{O}_{4}$ catalyst in the three nanotadpoles are nearly the same $(\sim 12 \%)$. 

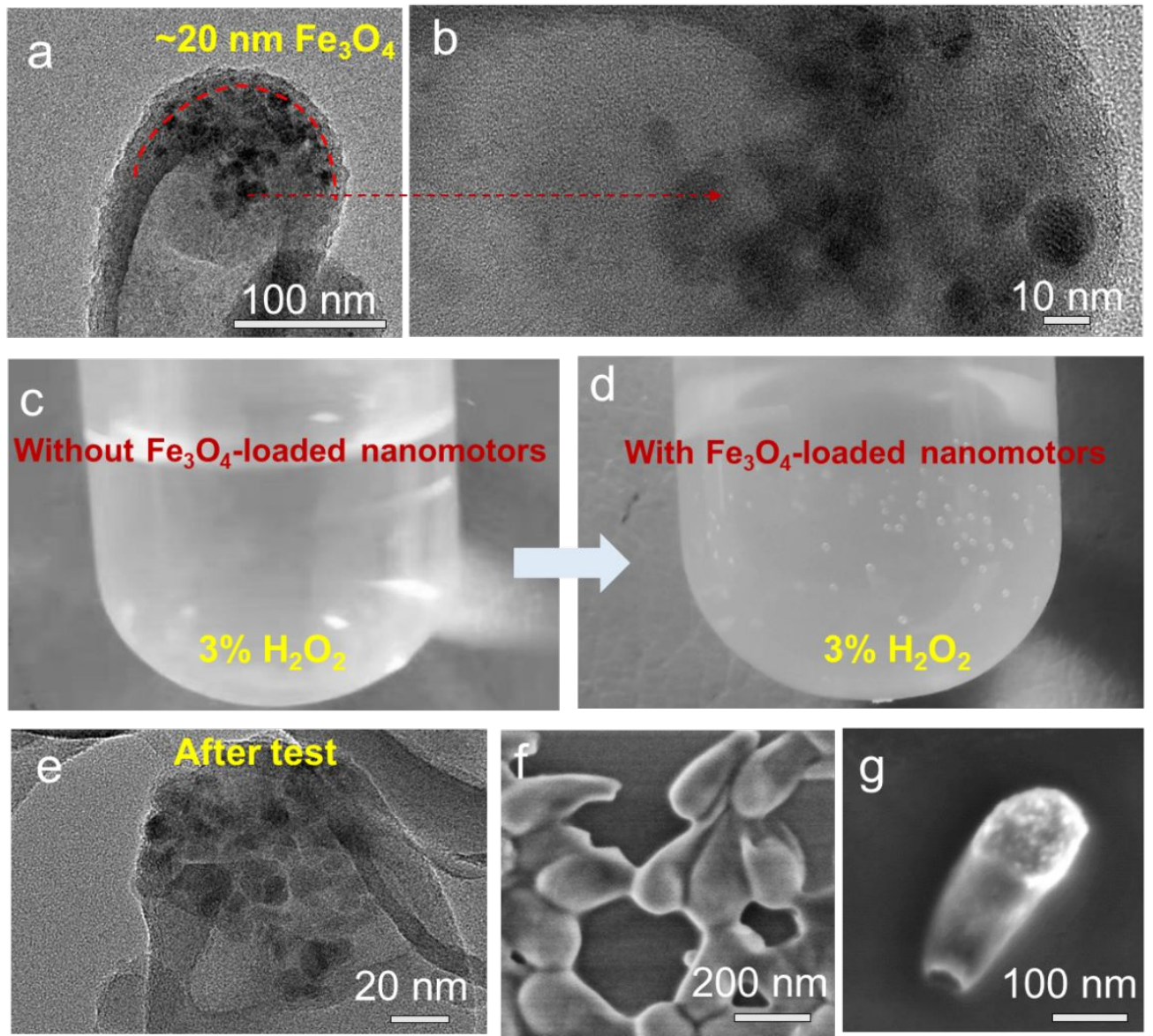

Figure S17. $(\mathrm{a}, \mathrm{b})$ TEM images of the $\mathrm{Fe}_{3} \mathrm{O}_{4}$ nanoparticles-loaded nanomotors with different magnifications; (c, d) Optical photos of the $\mathrm{H}_{2} \mathrm{O}_{2}$ solution $(3 \%$ ) before and after adding the $\mathrm{Fe}_{3} \mathrm{O}_{4}$-loaded nanomotors; (e) TEM and (f, g) SEM images of the $\mathrm{Fe}_{3} \mathrm{O}_{4}$-loaded nanomotors after several tests (in the testing solution for more than $2 \mathrm{~h}$ ). After adding the $\mathrm{Fe}_{3} \mathrm{O}_{4}$ nanoparticles-loaded nanomotors into the $\mathrm{H}_{2} \mathrm{O}_{2}$ solution (3\%), the bubbles can be clearly observed, indicating the generation of oxygen under the catalysis of $\mathrm{Fe}_{3} \mathrm{O}_{4}$ nanoparticles in the cavity of the nanotadpoles. After several rounds of the nanomotor tests, it can be seen that the $\mathrm{Fe}_{3} \mathrm{O}_{4}$ nanoparticles are still well-anchored at the head part of the cavity (not dispersed throughout the cavity), further illustrating the high stability of the $\mathrm{Fe}_{3} \mathrm{O}_{4}$ nanoparticles in the nanotadpoles. 

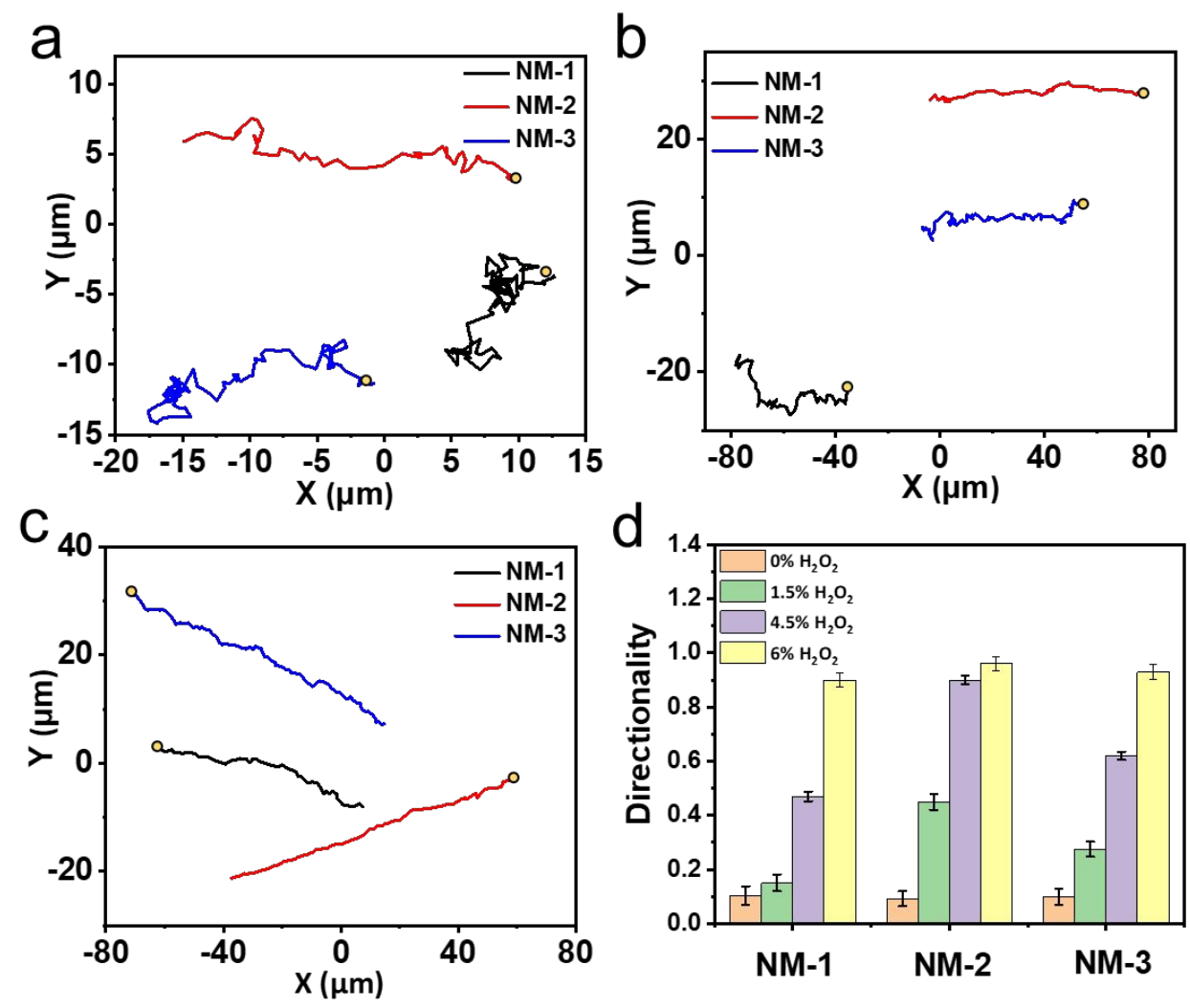

Figure S18. (a-c) Trajectory tracking of the nanomotors in different concentration of $\mathrm{H}_{2} \mathrm{O}_{2}$ solution in $10 \mathrm{~s}$; (a) $1.5 \%$, (b) $4.5 \%$; (c) $6 \%$. The tadpole-like mesoporous silica nanomotors exhibit a fuel-concentration-dependent diffusion property. (d) The corresponding ratio of the linear displacement to travel distance (defined as the directionality value) of the nanomotors in different concentration of $\mathrm{H}_{2} \mathrm{O}_{2}$ solution. The green circle in (a-c) represents the starting point of the trajectory. In the absence of $\mathrm{H}_{2} \mathrm{O}_{2}$, random motion of the nanomotors with different curvature is observed and no obvious difference in trajectory. Compared with NM-1 and NM-3, the directional movement trend of NM-2 motor with a curvature of $3.76 \times 10^{-2} \mathrm{~nm}^{-1}$ is more obvious, revealing that the curvature plays an important role in manipulating the motion behavior. We used the ratio between the linear-displacement and travel-distance (defined as the directionality value) to quantify the directionality of the nanomotors. The closer to 1 of this value is, the higher directionality of the nanomotor is. The results show that the directionality value of NM-2 motor is much closer to 1 (0.97) than that for NM-1 and NM-3, demonstrating a higher directionality of NM-2. 

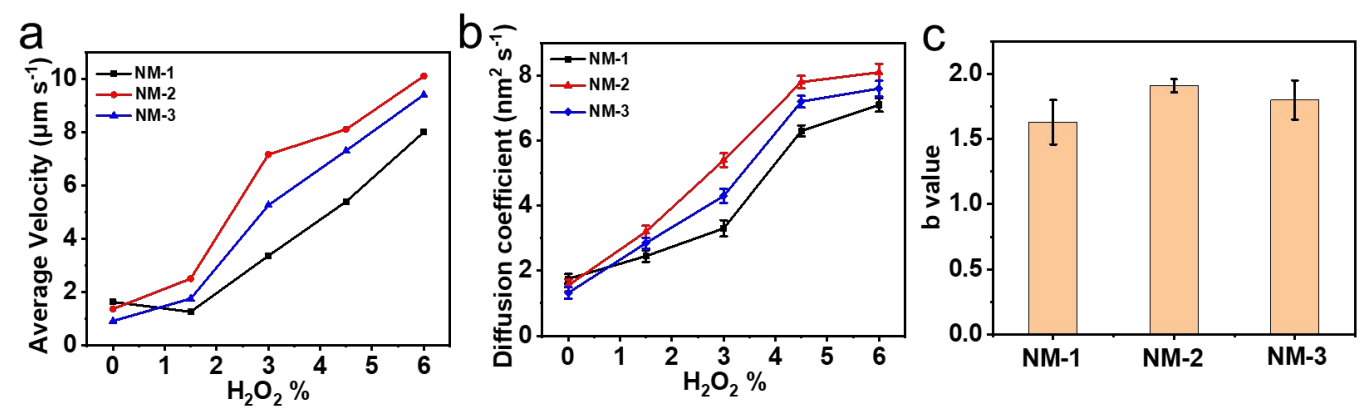

Figure S19. (a) Average velocity of the nanomotors calculated according to the travel distance of nanomotors, and (b) apparent diffusion coefficient values of the nanomotors calculated in different concentration of $\mathrm{H}_{2} \mathrm{O}_{2}$; (c) The anomalous diffusive exponent $(b)$ values of the nanomotors, which is calculated from the formula of $y=a x^{b}$ by simulating the MSD curves. It can be seen that all the three samples exhibit the similar average velocity and diffusion coefficient in the absence of $\mathrm{H}_{2} \mathrm{O}_{2}(0 \%)$, demonstrating that they possess the similar inherent physical properties (e.g. size and morphology). The velocities and apparent diffusion coefficients of the three nanomotors all show a significant growing trend with the increase of $\mathrm{H}_{2} \mathrm{O}_{2}$ concentration, but the growth rate of NM-2 nanomotor is more obvious, further indicating that the structural parameters can regulate the diffusion properties. Besides, by simulating the MSD curves according to the simulation formula of $y=a x^{b}$ (where the $b$ value determines the diffusion type of the motors, $b<1$, restricted diffusion; $1<b<2$, super diffusion; $b=2$, ballistic diffusion), the $b$ values of the motors with different curvatures are calculated. ${ }^{[3]}$ The anomalous diffusive exponent $(b)$ values for the motors are 1.63 (NM-1), 1.91 (NM-2), and 1.82 (NM-3), respectively. All the values show the type of super diffusion. Especially for $\mathrm{NM}-2$, its $b$ value is very close to 2 , indicating its super diffusion in the solution. 

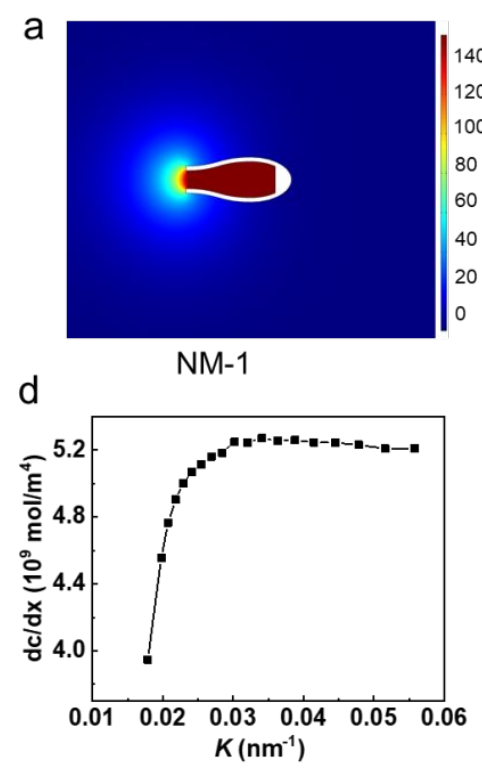

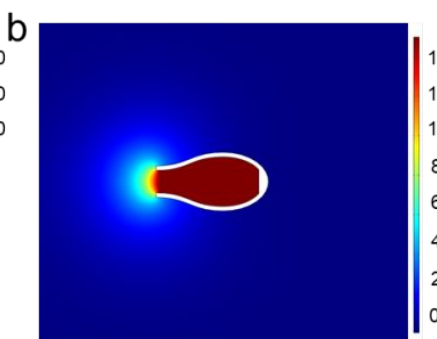

NM-2

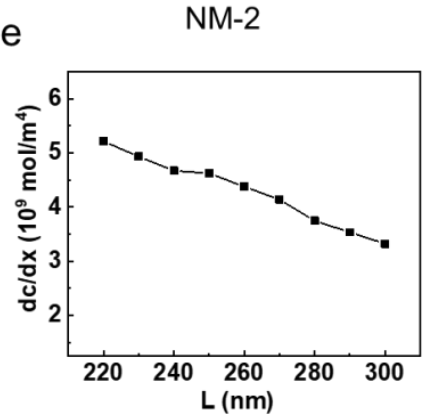

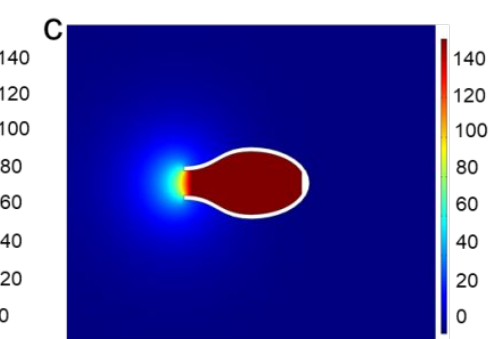

NM-3

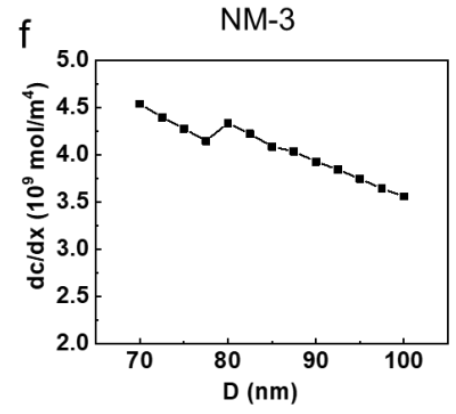

Figure S20. (a-c) The simulated steady-state concentration gradient distribution at the opening of the streamlined hollow nanoparticles with different structure parameters of head curvature, body length, opening size: (a) NM-1, (b) NM-2, and (c) NM-3; (d-f) The influence of the structure parameters of the nanomotors on concentration gradient at the opening of the streamlined hollow nanoparticles: (d) head curvature, (e) body length, and (f) opening diameter. Finite element analysis approach is used to evaluate the steady-state concentration gradient distribution at the opening of the streamlined hollow nanoparticles. It can be seen that a dissolved-oxygen diffusion flux is generated, leading to an efficient nanomotor propulsion. The steady-state concentration gradient is quite relevant to the structure parameters of the nanomotors (e.g. the head curvature, body length, opening size and so on). The concentration gradient increases first and then slows down as the curvature changes, and the curvature value of the NM-2 motor is just near the optimal condition. Combined with the influence of other structural parameters, NM-2 shows the optimal motion behavior. 

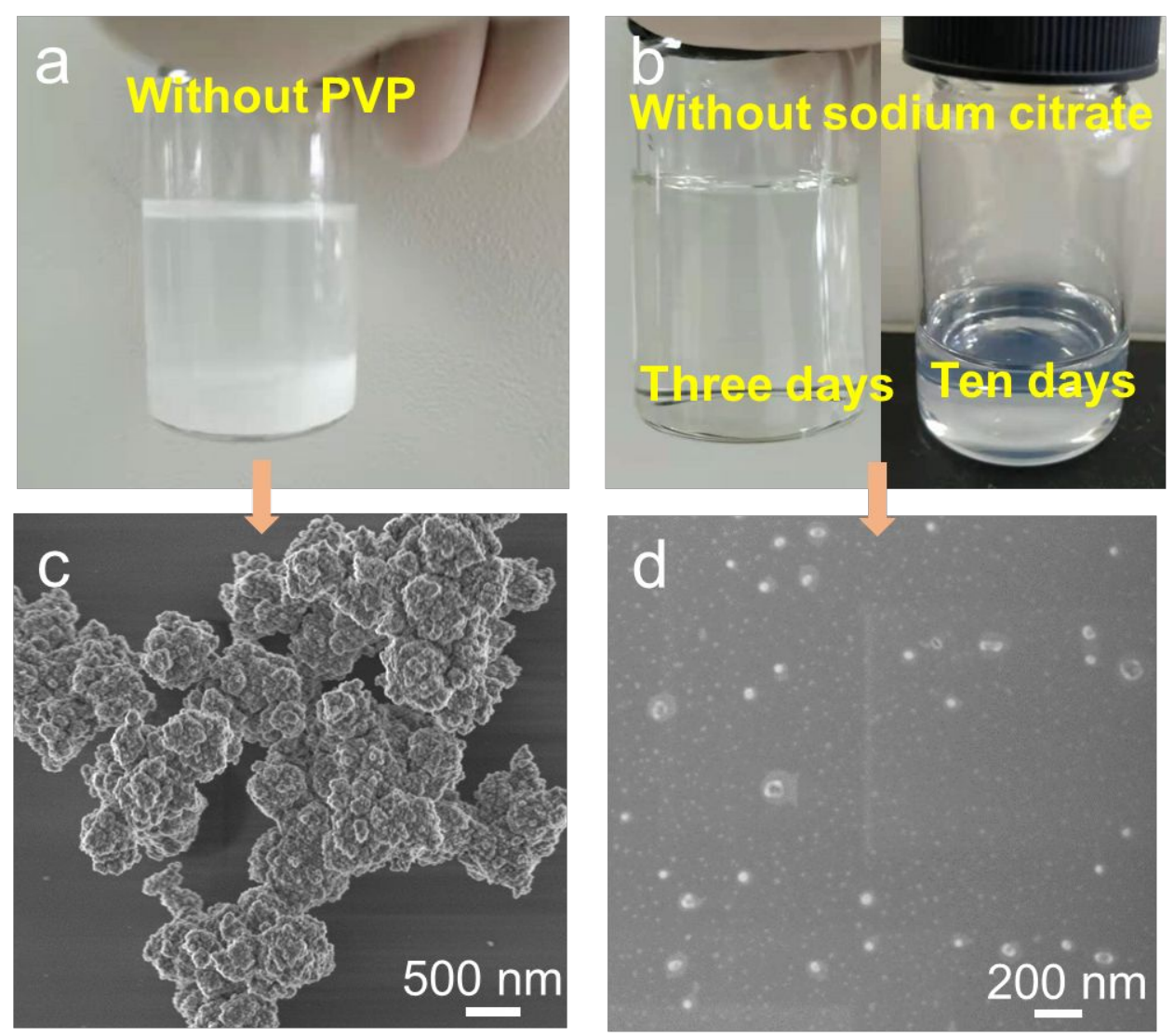

Figure S21. The influence of PVP and sodium citrate on the formation of the streamlined silica nanotadpoles. The optical photographs of the reaction solution with the absence of PVP (a) or sodium citrate (b), and the corresponding SEM images of the obtained nanoparticles (c, d). It is difficult to form the stable "water in oil" nanoemulsion without PVP, and further results in the phase separation of water and $n$ pentanol and the formation of aggregated nanoparticles with irregular morphology. Although a stable emulsion can be formed without sodium citrate, the hydrolysis rate of TEOS is extremely slow, and there is no product after three days of reaction. 
a

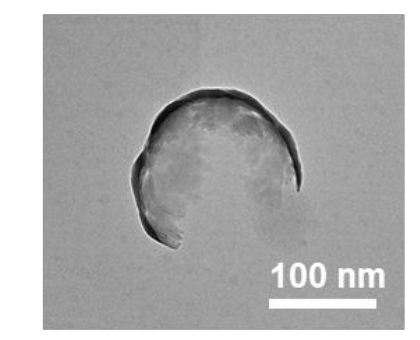

d

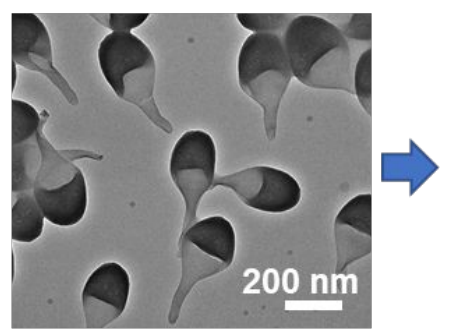

b

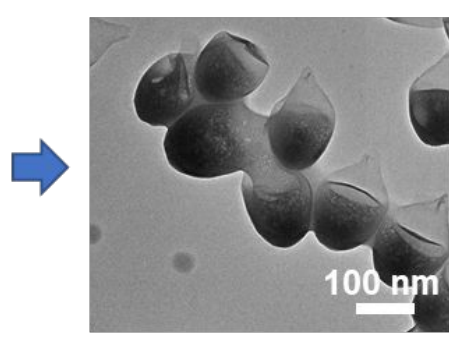

e

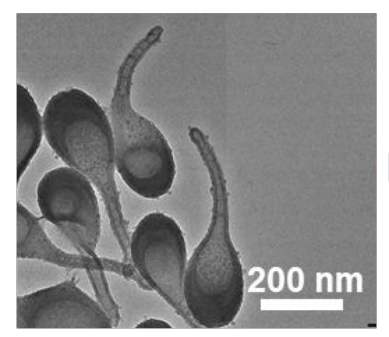

C

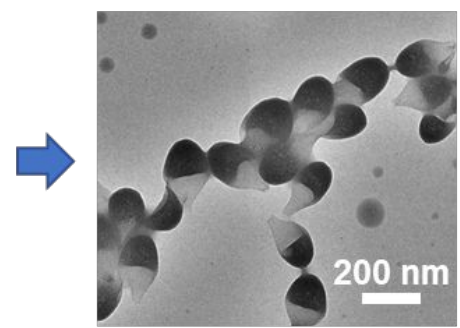

f

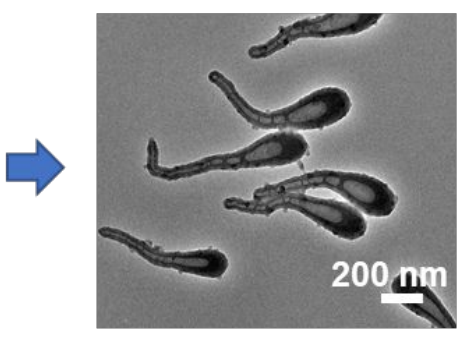

\section{Reaction time}

Figure S22. TEM images of the mesoporous silica nanotadpoles collected at different reaction times: (a) $20 \mathrm{~min}$; (b) $1.0 \mathrm{~h}$; (c) $1.5 \mathrm{~h}$; (d) $2.0 \mathrm{~h}$; (e) $3.0 \mathrm{~h}$; (f) $10 \mathrm{~h}$. It can be seen that a large bowl-shaped thin silica shell was formed within $0.5 \mathrm{~h}$. With the increase of the reaction time, the opening size of the nanoparticles gradually narrowed. The silica nanotadpoles with an opening size of $\sim 80 \mathrm{~nm}$ were obtained after $1.5 \mathrm{~h}$. When the reaction time further increased to $10 \mathrm{~h}$, the opening was closed, and the tadpoleshaped hollow nanoparticles with slender tails were formed. The head curvature of these streamlined mesoporous nanoparticles remains nearly constant $\left(3.76 \times 10^{-2} \mathrm{~nm}^{-1}\right)$ as the increase of reaction time. 


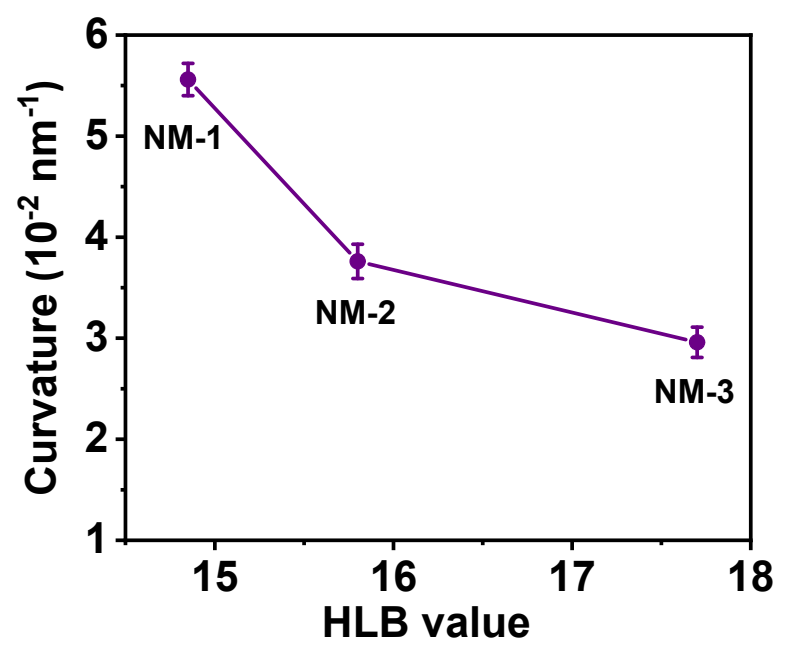

Figure S23. The correlation between the curvature and HLB values. With the increase of the HLB values for the surfactant templates, the curvatures of the obtained mesoporous nanoparticles are decreased from $5.56 \times 10^{-2}$ to $2.96 \times 10^{-2} \mathrm{~nm}^{-1}$. 


\section{References}

(1) Sahoo, Y.; Goodarzi, A.; Swihart, M. T.; Ohulchanskyy, T. Y.; Kaur, N.; Furlani, E. P.; Prasad, P. N. Aqueous ferrofluid of magnetite nanoparticles: fluorescence labeling and magnetophoretic control. J. Phys. Chem. B 2005, 109, 3879.

(2) Li, J. X.; Liu, W. J.; Wang, J. Y.; Rozen, I.; He, S.; Chen, C. R.; Kim, H. G.; Lee, H. J.; Lee, H. B. R.; Kwon, S. H.; Li, T. L.; Li, L. Q.; Wang, J.; Mei, Y. F. Nanoconfined atomic layer deposition of $\mathrm{TiO}_{2} / \mathrm{Pt}$ nanotubes: toward ultrasmall highly efficient catalytic nanorockets. Adv. Funct. Mater. 2017, 27, 1700598.

(3) Wan, M. M.; Wang, Q.; Li, X. Y.; Xu, B.; Fang, D.; Li, T.; Yu, Y. Q.; Fang, L. Y.; Wang, Y.; Wang, M.; Wang, F. H.; Mao, C.; Shen, J.; Wei, J. Systematic research and evaluation models of nanomotors for cancer combined therapy. Angew. Chem. Int. Ed. 2020, 59, 14458. 